\title{
S Research Square

\section{The Role of in-person Focus Groups in the Management of Urinary Incontinence in Women From a Mixed-methods, Randomized Pilot Study.}

Gina Toma ( $\square$ toma.gina212@gmail.com )

Mayo Clinic Arizona https://orcid.org/0000-0002-6872-8319

Alexandra Carolan

Division of Urology Mayo Clinic, Phoenix AZ

Skye Buckner-Petty

Department of Research Administration, Mayo Clinic Arizona

Laura Vargas

Department of Research Administration, Mayo Clinic Arizona

Christopher Wolter

Division of Urology Mayo Clinic, Phoenix AZ

\section{Aqsa Khan}

Division of Urology Mayo Clinic, Phoenix AZ

\section{Research Article}

Keywords: urinary incontinence (UI), focus group, social support, mixed methods

Posted Date: March 7th, 2022

DOI: https://doi.org/10.21203/rs.3.rs-1382799/v1

License: (1) This work is licensed under a Creative Commons Attribution 4.0 International License.

Read Full License 


\section{Abstract}

Purpose: To assess the quantitative and qualitative components of in-person focus groups as a potential intervention for female patients with urinary incontinence.

Methods: Women over the age of 18 seeking treatment for UI were randomized to standard care with focus group participation or to standard care alone. All participants completed validated questionnaires: MESA, UDI-6, OAB-SAT-q, PGI-S, PGI-I, SQoL-F, PHQ-9, IPAQ at the beginning and conclusion of the study. Questionnaires were analyzed with repeated measures of ANOVA models in an intention-to-treat manner. Three moderated focus group sessions were held and audio recorded. Recordings were transcribed and categorized by frequency into themes using grounded theory methodology.

Results: A total of 10 control and 8 intervention participants agreed to participate. Seven women attended all three focus group sessions and were included in the final analysis. Transcripts from focus group sessions observed women identified most with (1) urinary incontinence (UI) as a chronic disease, (2) shame managing $\mathrm{UI}$, and (3) social constraints of toileting. Participants self-reported appreciation of focus group participation and desire for on-going sessions. Analysis of the questionnaires did not demonstrate statistically significant differences.

Conclusion: Data ascertained from questionnaires was unable to demonstrate a meaningful effect in improved treatment outcomes for control and intervention participants. Grounded theory analysis of transcripts identified four primary themes: (1) appreciation of the focus group, (2) UI as a gendered issue, (3) lack of public awareness, and (4) history of negative provider interactions. All focus group participants self-reported interest in attending future focus group sessions.

\section{Background}

Urinary incontinence (UI) is a common lower urinary tract symptom (LUTS) experienced amongst men and women with prevalence increasing with age. ${ }^{1}$ Approximately $25 \%$ of women in the United States experience some form of $\mathrm{UI}$ with future rates predicted to rise from 28.1 million in 2010 to 43.8 million in 2050. ${ }^{2}$ Numerous studies have identified the impact of UI on the quality of life (QOL) in patients when compared to healthy and control group. ${ }^{3-5}$ The Quality-of-life Scale (QOLS) was created by American psychologist John Flanagan in the 1970 s to measure quality of life across patient groups and cultures. ${ }^{6}$ Since that time, there have been many studies examining the impact of urinary incontinence on patients' QOL. Mendes et. al reviewed 30 qualitative studies synthesizing the impact of UI on patients' lives and identified social support as an important component in the management of UI in women. ${ }^{7}$

Focus groups in various settings have demonstrated the ability to alleviate psychological and physiological distress in focus group participants. ${ }^{7}$ The exploration of focus groups is warranted to measure the potential benefit for patients with urinary incontinence. We aimed to assess the feasibility and acceptability of in-person focus groups as a potential intervention for female patients with urinary 
incontinence from a mixed methods approach. We conducted a mixed methods pilot study to investigate whether in-person focus group demonstrate meaningful significance in quantitative outcomes measured by validated questionnaires and qualitative outcomes analyzed from focus group transcripts and postsession surveys. This is an initial assessment to determine whether in-person focus groups for females with $\mathrm{UI}$ is strong enough to warrant further research and clinical implementation.

\section{Materials And Methods}

\section{Study Design}

In the Department of Urology and Female Reconstructive Surgery, we randomized women presenting for standard of care treatment for urinary incontinence (stress, urge, and mixed) into focus group sessions versus standard of care between April 2019 and June 2019 (Fig. 1). The study was approved by the Institutional Review Board (19-007977) at our institution.

\section{Study Population}

We assessed the eligibility of all women seeking UI treatment at our clinic who were 18 years or older, English-speaking, and had the ability to commute to the meeting facility without institutional assistance. We excluded women unable to attend all three focus group sessions $(n=1)$. All participants proceeded with the treatment of their urinary incontinence as decided with their provider; details on these varied treatments were not elicited by the research team. Patients were asked by their provider at the end of the clinic encounter if they would be willing to have a research coordinator speak to them about the study. If they were willing, the coordinator reviewed the study protocol and procedures with the patient. Participants were informed of the study duration, risks, and voluntary participation and then provided written informed consent. Demographics including age, race, and UI type were gathered by chart review (Table 1).

\section{Study Interventions}

Women were randomly assigned to attend in-person focus group sessions in conjunction with standard of care or standard of care alone with no focus group intervention. Three in-person focus group sessions were held in a private conference room at the treatment institution, occurring every two weeks from May 2019-June 2019. One member of the research team attended all three focus group sessions and served as the moderator. Using a script, the moderator welcomed and debriefed participants at the start and completion of each session. During each session, the moderator prompted group discussion of the management urinary incontinence. Predetermined questions were created by the research team to observe patient perspectives and feelings regarding the management of urinary incontinence [Appendix A]. Participant identification numbers were employed as a method of self-identification during group discussion. All three focus group sessions were audio-recorded and transcribed into a written deidentifiable document by the research team.

\section{Randomization}


Research coordinator randomly assigned eligible women using simple-randomization techniques. This approach consisted of an even number of cards labeled "control" $(n=10)$ and "intervention" $(n=10)$ that were pulled from a cup prior to research recruitment. All participants were randomly assigned deidentification numbers at the time of recruitment. Randomized patients continued standard of care for UI throughout study duration. The principal investigator and attending care teams were blinded to treatment allocation.

\section{Study Outcomes}

The primary outcome was to measure differences in pre and post questionnaires between control and focus group participants. All women completed 8 validated questionnaires: Medical, Epidemiological, and Social Aspects of Aging (MESA), Urinary Distress Inventory-6 (UDI-6), Overactive Bladder Satisfaction with Treatment Questionnaire (OAB-SAT-q), Patient Global Impression of Severity (PGI-S), Patient Global Impression of Improvement (PGI-I), Sexual Quality of Life-Female (SQoL-F), Patient Health Questionnaire (PHQ-9), and International Physical Activity Questionnaire (IPAQ) in order to assess differences between control and intervention participants. Questionnaires were administered to all participants at the time of consent and repeated 12 weeks later by telephone with the research coordinator. Validated questionnaires have been employed in urology evaluating continence outcomes, treatment satisfaction, and quality of life. ${ }^{8-10}$

A secondary outcome was to measure the feasibility and acceptability in continued focus group sessions. A written survey was administered at the beginning of each focus group session which incorporated open-ended warm-up questions regarding urinary incontinence and concluded with the assessment of the level of focus group comfort on a scale from 1-5, with 1 being "least comfortable" and 5 as "most comfortable". Participants were asked at the final focus group session to report how their experience could have been improved.

\section{Sample Size and Statistical Analysis}

Repeated measures ANOVA models were used to assess differences in pre and post questionnaire results administered twelve weeks apart between control and focus group participants. A sample size was not computed due to this being a pilot study.

To qualitatively analyze the session transcriptions, grounded theory methodology was used to ascertain innate themes addressed by focus group participants throughout all three sessions. An interview guide was created by the research team and implemented by the moderator during focus group sessions [Appendix A]. The investigator was not a trained focus group facilitator and prepared for sessions through the review of literature on moderation methods for focus groups. This method of qualitative analysis was first implemented in medical sociology by Barney Glaser and Anselm Straus. ${ }^{11}$ Glaser articulated 16 methodological principles encompassing the structural integrity of grounded theory methodology with qualitative data. Open Coding, Constant Comparative Method, Selective Coding, Delimiting and Memoing are Glaser principles which were applied to the audio recordings transcribed by 
the research team. Of these principles, Open Coding and Memoing were the most dominant principles applied in our qualitative analysis by four investigators on the research team. Investigators resolved differences in the interpretation of transcript thematic concepts through open discussion and modifying concepts until a consensus was reached.

\section{Results}

From April 2019-May 2019, 126 women seeking UI treatment at our institution's Urology department were eligible for study enrollment. Among these, 107 women declined for reasons including the following: schedule conflicts with focus group meeting times, lack of general interest in study participation, and/or travel constraints. The standard of care group initially consisted of 10 women, with 1 participant excluded due to lack of follow up, leaving a remainder of 9 control participants for analysis. Focus group participants originally consisted of 8 , with 1 participant excluded from analysis because they were unable to attend all focus group sessions, leaving a remainder of 7 focus group participants who attended all three sessions.

\section{Quantitative Questionnaire Results}

The validated questionnaires measured primary outcomes (MESA, UDI-6, OAB-SAT-q, PGI-S, PGI-I, SQoL-F, PHQ-9, IPAQ) and were analyzed with repeated measures of ANOVA models in an intention-to-treat manner. In the PHQ-9 results, the "level of difficulty performing daily tasks" differed between control and intervention participants $(p<0.05)$; control group participants reported performing tasks with less difficulty than focus group participants. When comparing the control to intervention groups, there were no statistically significant changes in pre to post intervention scores for any of the validated questionnaires.

\section{Qualitative Focus group Transcript Results}

Grounded theory methodology was used to assess innate themes within transcripts and surveys. Several dominant themes emerged from the focus group transcriptions. The most frequently identified codes identified during focus group discussion included references to UI as a chronic disease, "secrecy of condition," and social constraints of toileting, as seen in Table 2. "Secrecy of the condition" refers to the focus group participants' reported desire to keep their Ul a secret from their friends and family members.

The codes were synthesized to identify innate themes within the transcripts. Themes that emerged included an appreciation of focus group participation; urinary incontinence as a gendered issue; desire for comprehensive and personalized care; urinary incontinence causing shame, secrecy, and humiliation; lack of public awareness of diagnosis and treatment; the history of negative provider interactions; and preference for physician-led education. Representative quotes illustrating these themes are included in Table 3. Of these, the four most dominant themes included the following:

\section{Appreciation of focus group and continued interest in attending sessions}


Participants experienced solidarity after focus group attendance. Participants were appreciative of the opportunity to express their experience in managing UI with others. Moreover, the reported level of comfort remained constant throughout focus group sessions, with a self-reporting level of comfort averaging 4.5 out of 5 . The last focus group session concluded with a written free-response section for participants to share how their experience could have been improved. All participants noted additional focus group sessions would have been beneficial. $100 \%$ of participants $(n=8)$ were interested in attending additional sessions if offered in the future.

\section{Urinary incontinence as a gendered issue}

Women expressed their feelings that $\mathrm{Ul}$ affects women more severely than men in their personal and professional lives. Focus group participants believed UI management was more difficult to treat than male UI. More than half of the focus group participants preferred female urologists $(n=7)$ and believed male providers "lacked relatability" to female health challenges.

\section{Lack of public awareness of diagnosis and treatment}

Patients reflected on beliefs regarding the lack of public awareness of UI. Others discussed frustration about needing to teach family and friends about UI management. One participant contrasted the relative ease of breast cancer discussion with friends and family against the shame she felt discussing UI. Participants overwhelmingly believe more education regarding $\mathrm{UI}$ should be readily available to the public to decrease stigma and shame.

\section{History of negative provider interactions}

Focus group participants were prompted to share experiences managing $\mathrm{UI}$ in the healthcare setting. More than half of the focus group participants $(n=6)$ shared negative past experiences managing $\mathrm{UI}$ at previous health institutions. All focus group participants $(n=8)$ shared at least one negative experience with a urologist or gynecologist in UI management. Variations in the amount of education received from their provider regarding their diagnosis were expressed amongst focus group participants. Some participants $(n=4)$ recalled receiving educational pamphlets at some point in their healthcare journey and referred to them as informational. The remaining focus group participants $(n=4)$ did not recall receiving education pamphlets from providers. Participants were asked to identify an individual or provider they felt most comfortable discussing UI. $75 \%(n=6)$ patients identified their spouse, while the remaining $25 \%(n=$ 2) identified their provider.

\section{Discussion}

$\mathrm{UI}$ is a common condition, affecting approximately $25 \%$ of women in the United States, and rates are expected to continue rising in the setting of our aging population. ${ }^{2,3} \mathrm{UI}$ has been shown to negatively impact QOL measures for women. ${ }^{3}$ Two-thirds of women with UI report their quality of life is affected by their condition. ${ }^{12}$ Over time, patients with $\mathrm{Ul}$ experience decreases in QOL domains including physical, emotional, social functioning, bodily pain, vitality, mental health and general health. ${ }^{3}$ As providers 
continue to care for women with Ul, it is crucial to identify mechanisms to offer support and hope for women with this condition if we aim to improve QOL.

Qualitative research has identified "forming a circle of trust" to be a strategy employed by patients to deal with $\mathrm{UI} .{ }^{11}$ In a meta-ethnography analysis of 41 studies on urinary incontinence, investigators identified secrecy and shame surrounding urinary incontinence to be a barrier to care which prevents patients from focusing on strategies to improve their continence. ${ }^{11}$ This raises the possibility that structured focus groups may provide patients a safe, confidential setting in which to discuss their condition and improve their QOL. In other medical settings, focus groups have been shown to improve QOL. For example, selfhelp group participation among breast cancer survivors was shown to improve the patients' sense of empowerment and QOL. ${ }^{8}$ In a qualitative study of patients with rheumatoid arthritis, participants in a focus group reported improvements in QOL associated with increased knowledge and self-efficacy. ${ }^{9}$

Our mixed-methods study therefore aimed to evaluate whether in-person focus group participation could reduce UI symptoms and improve quality of life as measured quantitatively by validated questionnaires and qualitatively by extracting themes by grounded theory analysis of focus group transcripts. Data obtained from validated questionnaires were not able to demonstrate improved treatment outcomes in women with urinary incontinence. Despite quantitative results, the themes drawn from grounded theory analysis from focus group transcripts suggest social focus groups may have the potential to improve quality of life for patients with UI.

The four dominant themes identified with our qualitative analysis of focus group transcripts were the following: lack of public awareness of diagnosis and treatment, $\mathrm{UI}$ as a gendered issue, a history of negative provider interactions, and an appreciation of the focus group with continued interest in attending sessions. The themes overall align with previous findings in the literature. A systematic review by Mendes et al., identified common themes related to how women experience Ul such as isolation and secrecy surrounding $\mathrm{UI}$ within social groups, overall lack of patient education and understanding of $\mathrm{UI}$ as a chronic condition, and the overarching generalization of shame regarding the condition. ${ }^{13}$ The dominant theme extracted from the transcripts in our study regarding continued interest in attending further sessions adds to previous findings regarding social support as an important component of coping with UI. Despite quantitative results, this theme suggests focus groups may have the potential to improve quality of life for patients with UI.

\section{Limitations:}

There were several limitations to this study. Social desirability bias may have impacted quantitative analysis of data ascertained by validated questionnaires. Challenges existed during the recruitment phase of focus group participants notably due to time constraints of pre-determined meeting times. Those who participated in focus group intervention were elderly and mostly retired except for one participant that worked part-time. This may impact the generalizability of the results. Our small sample size may have limited the ability to identify significant relationships between groups as well as the short 
follow-up time. Further studies with randomization, larger participant numbers, and longer follow-up will help to assuage these limitations.

\section{Conclusion}

To date, this is the first study to evaluate validated questionnaires and focus groups to measure QoL in women with urinary incontinence. The results indicate minor differences in validated questionnaires and desire for continued focus group participation.

\section{Declarations}

Ethics approval: All procedures performed in studies involving human participants were in accordance with the ethical standards of the institutional and/or national research committee and with the 1964 Helsinki Declaration and its later amendments or comparable ethical standards. The study was approved by the Mayo Clinic- Institutional Review Board (19-007977).

Consent to Participate: Written informed consent was obtained from all individual participants included in the study.

Consent for publication: Not applicable.

Availability of data and materials: All data generated or analyzed during this study are included in this published article [and its supplementary information files].

Competing interests: All authors certify that they have no affiliations with or involvement in any organization or entity with any financial interest or non-financial interest in the subject matter or materials discussed in this manuscript.

Funding: Not applicable.

Authors' Contribution: Gina Toma: Protocol/project development, data collection and management, data analysis, manuscript writing/editing, focus group moderator

Alexandra Carolan: Data collection and management, data analysis, manuscript writing/editing

Skye Buckner-Petty: Data analysis, manuscript writing/editing

Laura Vargas: Data analysis, manuscript writing/editing

Christopher Wolter: Manuscript writing/editing

Aqsa Khan: Protocol/project development, data analysis, manuscript writing/editing

Acknowledgments: Not applicable. 


\section{References}

1. Wu, J. M., Vaughan, C. P., Goode, P. S., Redden, D. T., Burgio, K. L., Richter, H. E., \& Markland, A. D. (2014). Prevalence and Trends of Symptomatic Pelvic Floor Disorders in U.S. Women. Obstetrics and Gynecology. 2014:123:141-148. http://doi.org/10.1097/AOG.0000000000000057

2. Wu JMHA, Fulton RG, Myers ER. Forecasting the prevalence of pelvic floor disorders in U.S. Women: 2010-2050. Obstet Gynecol. 2009; 114:1278-1283.

3. Hagglund D, Walker-Engstrom ML, Larsson G, et al. Changes in urinary incontinence and quality of life after four years. A population-based study of women aged 22-50 years. Scand J Prim Health Care. 2004;22:112-117.

4. Coyne KS, Payne C, Bhattacharyya SK, et al. The impact of urinary urgency and frequency on healthrelated quality of life in overactive bladder: results from a national community survey. Value Health. 2004;7:455-463.

5. Oh SJ, Ku JH. Impact of stress urinary incontinence and overactive bladder on micturition patterns and health-related quality of life. Int Urogynecol J Pelvic Floor Dysfunct. 2007;18:65-71.

6. Bartoli, S., Aguzzi, G., \& Tarricone, R. (2010). Impact on quality of life of urinary incontinence and overactive bladder: a systematic literature review. Urology. 2010;75(3): 491-500.

7. Nikchols, K., \& Jenkinson, J. (2006). Leading a focus group: a practical guide. Open University Press. 2006; 23-24 Shin S, Park H. (2017). Effect of empowerment on the quality of life of the survivors of breast cancer: The moderating effect of self-help group participation. Jpn J Nurs Sci. 14(4):311-319. doi:10.1111/jjns. 12161

8. Shin S, Park H. (2017). Effect of empowerment on the quality of life of the survivors of breast cancer: The moderating effect of self-help group participation. Jpn J Nurs Sci. 14(4):311-319. doi:10.1111/jjns.12161

9. McCarron A. An exploration of the perceived effects of a focus group for individuals with rheumatoid arthritis (2015). J Am Assoc Nurse Pract. 27(3):160-166. doi:10.1002/2327-6924.12146

10. Toye, F., \& Barker, K. L. (2020). A meta-ethnography to understand the experience of living with urinary incontinence: 'is it just part and parcel of life?'. BMC urology, 20(1), 1. https://doi.org/10.1186/s12894-019-0555-4

11. Hayder, D., \& Schnepp, W. (2010). Experiencing and managing urinary incontinence: a qualitative study. Western Journal of Nursing Research, 32(4), 480-496.

12. Temml C, Haidinger G, Scmidbauer J, Schatzl G, Madersbacher S. Urinary Incontinence in both sexes: prevalence rates and impact on quality of life and sexual life. Neurourol Urodynam. 2000;19(3):25971.

13. Mendes, A., Hoga, L., Gonçalves, B., Silva, P., \& Pereira, P. (2017). Adult women's experiences of urinary incontinence: a systematic review of qualitative evidence. JBI database of systematic reviews and implementation reports, 15(5), 1350-1408 


\section{Tables}

Table 1 Demographic Table

Table 4 Characteristics of study population

\begin{tabular}{|llll|}
\hline Variables & $\begin{array}{l}\text { Intervention } \\
(\mathbf{N}=\mathbf{8})\end{array}$ & $\begin{array}{l}\text { Control } \\
(\mathbf{N}=9)\end{array}$ & P Value \\
\hline Age in years & & & $<.0001$ \\
\hline Mean \pm SD & $63.1 \pm 11.3$ & $71.8 \pm 9.32$ & \\
Min-Max & $41-77$ & $52-84$ & \\
Median & 66 & 74 & \\
\hline Gender & & & $<.0001$ \\
\hline Female & $8(100)$ & $9(100)$ & \\
Male & $0(0)$ & $0(0)$ & \\
\hline Race & & & $<.0001$ \\
\hline Asian & $1(12.0)$ & $0(0)$ & \\
African American & $1(12.0)$ & $0(0)$ & \\
White & $6(76.0)$ & $9(100)$ & \\
\hline Ul Type & & & $<.0001$ \\
\hline Stress & $0(0)$ & $2(22.0)$ & \\
Urge & $5(62.0)$ & $6(67.0)$ & \\
Mixed & $3(38.0)$ & $0(0)$ & $1(11.0)$ \\
Other & $0(0)$ & & \\
\hline
\end{tabular}

Table 2 Transcript memo frequencies from support group sessions 1, 2, 3 


\begin{tabular}{|ll|}
\hline Transcript Memo: & Sum of Frequency (n) \\
\hline Reference of UI as a chronic disease & 10 \\
Secrecy of condition & 8 \\
Social constraints of toileting & 7 \\
Self-teaching & 6 \\
Poor recollection of treatment plan & 6 \\
"I had no idea this was a thing" & 6 \\
Interference with daily living & 5 \\
Preference for female provider & 5 \\
Preference for verbal teaching & 4 \\
Belief of bias information & 4 \\
Confusion of diagnosis & 4 \\
Negative forecasting of prognosis & 4 \\
Unanticipated Ul recurrence & 4 \\
Respect for physician knowledge & 3 \\
Self-directed pursuit of treatment & 3 \\
Internet distrust & 3 \\
Preference for personalized care & 3 \\
Dietary modifications & 3 \\
Desire to resume physical exercise & 2 \\
Reference to pediatric incontinence & 2 \\
Desire of new insight and new therapies & 2 \\
Fragmented specialized care & 2 \\
Recommendation of Kegels & 2 \\
Preference of UI literature & 100 \\
\hline Grand Total & \\
\hline
\end{tabular}

Table 3 Innate themes and quotes from support group participants 


\begin{tabular}{|ll|}
\hline Theme & Representative Quotes \\
\hline $\begin{array}{l}\text { Appreciation of } \\
\text { Support Group }\end{array}$ & "I would say I probably said more here than I have ever said before." \\
"I had not discussed my situation managing urinary incontinence so \\
thoroughly before this study"
\end{tabular}

\section{Appendix}

Appendix A is not available with this version.

\section{Figures}

\section{Image not available with this version}

\section{Figure 1}

This image is not available with this version. 